\title{
PHASE TRANSITIONS FOR A MODEL WITH UNCOUNTABLE SET OF SPIN VALUES ON A CAYLEY TREE
}

\author{
YU. KH. ESHKABILOV, U. A. ROZIKOV, G.I. BOTIROV
}

\begin{abstract}
In this paper we consider a model with nearest-neighbor interactions and with the set $[0,1]$ of spin values, on a Cayley tree of order $k \geq 2$. To study translationinvariant Gibbs measures of the model we drive an nonlinear functional equation. For $k=2$ and 3 under some conditions on parameters of the model we prove non-uniqueness of translation-invariant Gibbs measures (i.e. there are phase transitions).
\end{abstract}

\author{
Mathematics Subject Classifications (2010). 82B05, 82B20 (primary); 60K35 \\ (secondary) \\ Key words. Cayley tree, configuration, Gibbs measures, phase transitions.
}

\section{INTRODUCTION}

A central problem in the theory of Gibbs measures is to describe infinite-volume (or limiting) Gibbs measures corresponding to a given Hamiltonian.

In order to study the phase transition (Gibbs measures) problem for a system on $Z^{d}$ and on Cayley tree there are two different methods: Pirogov-Sinai theory on $Z^{d}$, Markov random field theory and recurrent equations of this theory on Cayley tree.

The papers [1]- [5], [8], [9]- [10], [13], [14], [15] are devoted to models with a finite set of spin values. It were shown that these models have finitely many translation-invariant and uncountable numbers of the non-translation-invariant extreme Gibbs measures. Also for several models (see, for example, [6, 8, 10]) it were proved that there exist three periodic Gibbs measures (which are invariant with respect to normal subgroups of finite index of the group representation of the Cayley tree) and there are uncountable number of non-periodic Gibbs measures.

In [7] the Potts model with a countable set of spin values on a Cayley tree is considered and it was showed that the set of translation-invariant splitting Gibbs measures of the model contains at most one point, independently on parameters of the Potts model with countable set of spin values on the Cayley tree. This is a crucial difference from the models with a finite set of spin values, since the last ones may have more than one translation-invariant Gibbs measures.

This paper is continuation of our investigations [3], 4], [11. In [11] models (Hamiltonians) with nearest-neighbor interactions and with the (uncountable) set $[0,1]$ of spin values, on a Cayley tree $\Gamma^{k}$ of order $k \geq 1$ were studied.

We reduced the problem to the description of the solutions of some nonlinear integral equation. Then for $k=1$ we showed that the integral equation has a unique solution. 
In case $k \geq 2$ some models (with the set $[0,1]$ of spin values) which have a unique splitting Gibbs measure are constructed. In our next paper [3] it was found a sufficient condition on Hamiltonian of the model with an uncountable set of spin values under which the model has unique translation-invariant splitting Gibbs measure. In [4] we have constructed several examples of models with uncountable set of spin values which have phase transitions.In this paper we shall construct new models with nearest-neighbor interactions and with the set $[0,1]$ of spin values, on a Cayley tree order $k$. We prove that each of the constructed model has a phase transition.

\section{Preliminaries}

A Cayley tree $\Gamma^{k}=(V, L)$ of order $k \geq 1$ is an infinite homogeneous tree, i.e., a graph without cycles, with exactly $k+1$ edges incident to each vertices. Here $V$ is the set of vertices and $L$ that of edges (arcs).

Consider models where the spin takes values in the set $[0,1]$, and is assigned to the vertexes of the tree. For $A \subset V$ a configuration $\sigma_{A}$ on $A$ is an arbitrary function $\sigma_{A}: A \rightarrow[0,1]$. Denote $\Omega_{A}=[0,1]^{A}$ the set of all configurations on $A$. A configuration $\sigma$ on $V$ is then defined as a function $x \in V \mapsto \sigma(x) \in[0,1]$; the set of all configurations is $[0,1]^{V}$. We consider the (formal) Hamiltonian of the model is :

$$
H=H_{\theta, \beta}(\sigma)=-\sum_{\langle x, y\rangle \in L} \xi_{\sigma(x) \sigma(y)}(\theta, \beta), \quad \sigma \in \Omega_{A}
$$

where $\theta \in R$ is a coupling constant, $\beta=\frac{1}{T}, T>0$ is temperature and $\xi:(u, v) \in$ $[0,1]^{2} \rightarrow \xi_{u v} \in R$ is a given bounded, measurable function. As usually, $\langle x, y\rangle$ stands for nearest neighbor vertices.

Note that, a Gibbs measures for the model (2.1) with $\xi_{t, u}(J, \beta):=J \xi_{t, u}$ are investigated in [3, [4, 11].

Let $\lambda$ be the Lebesgue measure on $[0,1]$. On the set of all configurations on $A$ the a priori measure $\lambda_{A}$ is introduced as the $|A|$ fold product of the measure $\lambda$. Here and further on $|A|$ denotes the cardinality of $A$. We consider a standard sigma-algebra $\mathcal{B}$ of subsets of $\Omega=[0,1]^{V}$ generated by the measurable cylinder subsets. A probability measure $\mu$ on $(\Omega, \mathcal{B})$ is called a Gibbs measure (with Hamiltonian $H$ ) if it satisfies the DLR equation, namely for any $n=1,2, \ldots$ and $\sigma_{n} \in \Omega_{V_{n}}$ :

$$
\mu\left(\left\{\sigma \in \Omega:\left.\sigma\right|_{V_{n}}=\sigma_{n}\right\}\right)=\int_{\Omega} \mu(\mathrm{d} \omega) \nu_{\left.\omega\right|_{W_{n+1}}}^{V_{n}}\left(\sigma_{n}\right)
$$

where $\nu_{\left.\omega\right|_{W_{n+1}}}^{V_{n}}$ is the conditional Gibbs density

$$
\nu_{\left.\omega\right|_{W_{n+1}}}^{V_{n}}\left(\sigma_{n}\right)=\frac{1}{Z_{n}\left(\left.\omega\right|_{W_{n+1}}\right)} \exp \left(-\beta H\left(\sigma_{n} \|\left.\omega\right|_{W_{n+1}}\right)\right) .
$$

Here and below, $W_{l}$ stands for a 'sphere' and $V_{l}$ for a 'ball' on the tree, of radius $l=1,2, \ldots$, centered at a fixed vertex $x^{0}$ (an origin):

$$
W_{l}=\left\{x \in V: d\left(x, x^{0}\right)=l\right\}, \quad V_{l}=\left\{x \in V: d\left(x, x^{0}\right) \leq l\right\}
$$


and

$$
L_{n}=\left\{\langle x, y\rangle \in L: x, y \in V_{n}\right\} ;
$$

distance $d(x, y), x, y \in V$, is the length of (i.e. the number of edges in) the shortest path connecting $x$ with $y . \Omega_{V_{n}}$ is the set of configurations in $V_{n}$ (and $\Omega_{W_{n}}$ that in $W_{n}$; see below). Furthermore, $\left.\sigma\right|_{V_{n}}$ and $\left.\omega\right|_{W_{n+1}}$ denote the restrictions of configurations $\sigma, \omega \in \Omega$ to $V_{n}$ and $W_{n+1}$, respectively. Next, $\sigma_{n}: x \in V_{n} \mapsto \sigma_{n}(x)$ is a configuration in $V_{n}$ and $H\left(\left.\sigma_{n}|| \omega\right|_{W_{n+1}}\right)$ is defined as the sum $H\left(\sigma_{n}\right)+U\left(\sigma_{n},\left.\omega\right|_{W_{n+1}}\right)$ where

$$
\begin{aligned}
H\left(\sigma_{n}\right) & =-\sum_{\langle x, y\rangle \in L_{n}} \xi_{\sigma_{n}(x) \sigma_{n}(y)}, \\
U\left(\sigma_{n},\left.\omega\right|_{W_{n+1}}\right) & =-\sum_{\langle x, y\rangle: x \in V_{n}, y \in W_{n+1}} \xi_{\sigma_{n}(x) \omega(y)} .
\end{aligned}
$$

Finally, $Z_{n}\left(\left.\omega\right|_{W_{n+1}}\right)$ stands for the partition function in $V_{n}$, with the boundary condition $\left.\omega\right|_{W_{n+1}}$ :

$$
Z_{n}\left(\left.\omega\right|_{W_{n+1}}\right)=\int_{\Omega_{V_{n}}} \exp \left(-\beta H\left(\widetilde{\sigma}_{n} \|\left.\omega\right|_{W_{n+1}}\right)\right) \lambda_{V_{n}}\left(d \widetilde{\sigma}_{n}\right) .
$$

Due to the nearest-neighbor character of the interaction, the Gibbs measure possesses a natural Markov property: for given a configuration $\omega_{n}$ on $W_{n}$, random configurations in $V_{n-1}$ (i.e., 'inside' $\left.W_{n}\right)$ and in $V \backslash V_{n+1}$ (i.e., 'outside' $W_{n}$ ) are conditionally independent.

We use a standard definition of a translation-invariant measure (see, e.g., [12]). The main object of study in this paper are translation-invariant Gibbs measures for the model (2.1) on Cayley tree.

Write $x<y$ if the path from $x^{0}$ to $y$ goes through $x$. Call vertex $y$ a direct successor of $x$ if $y>x$ and $x, y$ are nearest neighbors. Denote by $S(x)$ the set of direct successors of $x$. Observe that any vertex $x \neq x^{0}$ has $k$ direct successors and $x^{0}$ has $k+1$.

Let $h: x \in V \mapsto h_{x}=\left(h_{t, x}, t \in[0,1]\right) \in R^{[0,1]}$ be mapping of $x \in V \backslash\left\{x^{0}\right\}$. Given $n=1,2, \ldots$, consider the probability distribution $\mu^{(n)}$ on $\Omega_{V_{n}}$ defined by

$$
\mu^{(n)}\left(\sigma_{n}\right)=Z_{n}^{-1} \exp \left(-\beta H\left(\sigma_{n}\right)+\sum_{x \in W_{n}} h_{\sigma(x), x}\right),
$$

Here, as before, $\sigma_{n}: x \in V_{n} \mapsto \sigma(x)$ and $Z_{n}$ is the corresponding partition function:

$$
Z_{n}=\int_{\Omega_{V_{n}}} \exp \left(-\beta H\left(\widetilde{\sigma}_{n}\right)+\sum_{x \in W_{n}} h_{\widetilde{\sigma}(x), x}\right) \lambda_{V_{n}}\left(d \widetilde{\sigma}_{n}\right) .
$$

The probability distributions $\mu^{(n)}$ are compatible if for any $n \geq 1$ and $\sigma_{n-1} \in \Omega_{V_{n-1}}$ :

$$
\int_{\Omega_{W_{n}}} \mu^{(n)}\left(\sigma_{n-1} \vee \omega_{n}\right) \lambda_{W_{n}}\left(d\left(\omega_{n}\right)\right)=\mu^{(n-1)}\left(\sigma_{n-1}\right) .
$$


Here $\sigma_{n-1} \vee \omega_{n} \in \Omega_{V_{n}}$ is the concatenation of $\sigma_{n-1}$ and $\omega_{n}$. In this case there exists a unique measure $\mu$ on $\Omega_{V}$ such that, for any $n$ and $\sigma_{n} \in \Omega_{V_{n}}, \mu\left(\left\{\left.\sigma\right|_{V_{n}}=\sigma_{n}\right\}\right)=$ $\mu^{(n)}\left(\sigma_{n}\right)$.

Definition 2.1. The measure $\mu$ is called splitting Gibbs measure corresponding to Hamiltonian (2.1) and function $x \mapsto h_{x}, x \neq x^{0}$.

The following statement describes conditions on $h_{x}$ guaranteeing compatibility of the corresponding distributions $\mu^{(n)}\left(\sigma_{n}\right)$.

Proposition 2.2. [11] The probability distributions $\mu^{(n)}\left(\sigma_{n}\right), n=1,2, \ldots$, in (2.2) are compatible iff for any $x \in V \backslash\left\{x^{0}\right\}$ the following equation holds:

$$
f(t, x)=\prod_{y \in S(x)} \frac{\int_{0}^{1} \exp \left(\beta \xi_{t u}\right) f(u, y) d u}{\int_{0}^{1} \exp \left(\beta \xi_{0 u}\right) f(u, y) d u} .
$$

Here, and below $f(t, x)=\exp \left(h_{t, x}-h_{0, x}\right), t \in[0,1]$ and $d u=\lambda(d u)$ is the Lebesgue measure.

From Proposition 2.2 it follows that for any $h=\left\{h_{x} \in R^{[0,1]}, \quad x \in V\right\}$ satisfying (2.5) there exists a unique Gibbs measure $\mu$ and vice versa. However, the analysis of solutions to (2.5) is not easy. This difficulty depends on the given function $\xi$.

Let $\xi_{t u}$ is a continuous function and we are going to construct functions $\xi_{t u}$ under which the equation (2.5) has at least two solutions in the class of translational-invariant functions $f(t, x)$, i.e $f(t, x)=f(t)$, for any $x \in V$. For such functions equation (2.5) can be written as

$$
f(t)=\left(\frac{\int_{0}^{1} K(t, u) f(u) d u}{\int_{0}^{1} K(0, u) f(u) d u}\right)^{k},
$$

where $K(t, u)=\exp \left(\beta \xi_{t u}\right), f(t)>0, t, u \in[0,1]$.

We put

$$
C^{+}[0,1]=\{f \in C[0,1]: f(x) \geq 0\} .
$$

We are interested to positive continuous solutions to (2.6).

\section{A Representation of solutions}

For every $k \in \mathbb{N}$ we consider an integral operator $H_{k}$ acting in the cone $C^{+}[0,1]$ as

$$
\left(H_{k} f\right)(t)=\int_{0}^{1} K(t, u) f^{k}(u) d u, \quad k \in \mathbb{N} .
$$

The operator $H_{k}$ is called Hammerstein's integral operator of order $k$. Clearly that, if $k \geq 2$ then $H_{k}$ is a nonlinear operator.

It is known that the set of translational invariant Gibbs measures of the model (2.1) is described by the fixed points of the Hammerstein's operator ( [11]). 
Let $k \geq 2$ in the model $(2.1)$ and

$$
\xi_{t, u}=\xi_{t, u}(\theta, \beta)=\frac{1}{\beta} \ln \left(1+\theta \sqrt[3]{4\left(t-\frac{1}{2}\right)\left(u-\frac{1}{2}\right)}\right), \quad t, u \in[0,1]
$$

where $0 \leq \theta<1$. Then for the kernel $K(t, u)$ of the Hammerstein's operator $H_{k}$ we have

$$
K(t, u)=1+\theta \sqrt[3]{4\left(t-\frac{1}{2}\right)\left(u-\frac{1}{2}\right)} .
$$

We define the operator $V_{k}:(x, y) \in R^{2} \rightarrow\left(C_{1}, C_{2}\right) \in R^{2}$ by

$$
V_{k}:\left\{\begin{array}{l}
x^{\prime}=3\left(\frac{(x+y \theta \sqrt[3]{2})^{k+1}-(x-y \theta \sqrt[3]{2})^{k+1}}{2 \sqrt[3]{2}(k+1) y \theta}-\frac{(x+y \theta \sqrt[3]{2})^{k+2}+(x-y \theta \sqrt[3]{2})^{k+2}}{\sqrt[3]{4}(k+1)(k+2) y^{2} \theta^{2}}+\right. \\
\left.+\frac{(x+y \theta \sqrt[3]{2})^{k+3}-(x-y \theta \sqrt[3]{2})^{k+3}}{2(k+1)(k+2)(k+3) y^{3} \theta^{3}}\right) \\
y^{\prime}=3\left(\frac{(x+y \theta \sqrt[3]{2})^{k+1}+(x-y \theta \sqrt[3]{2})^{k+1}}{2 \sqrt[3]{4}(k+1) y \theta}-\frac{3\left((x+y \theta \sqrt[3]{2})^{k+2}-(x-y \theta \sqrt[3]{2})^{k+2}\right)}{4(k+1)(k+2) y^{2} \theta^{2}}+\right. \\
\left.+\frac{3\left((x+y \theta \sqrt[3]{2})^{k+3}+(x-y \theta \sqrt[3]{2})^{k+3}\right)}{2 \sqrt[3]{2}(k+1)(k+2)(k+3) y^{3} \theta^{3}}-\frac{3\left((x+y \theta \sqrt[3]{2})^{k+4}-(x-y \theta \sqrt[3]{2})^{k+4}\right)}{2 \sqrt[3]{4}(k+1)(k+2)(k+3)(k+4) y^{4} \theta^{4}}\right)
\end{array}\right.
$$

Proposition 3.1. A function $\varphi \in C[0,1]$ is a solution of the Hammerstein's equation

$$
\left(H_{k} f\right)(t)=f(t)
$$

iff $\varphi(t)$ has the following form

$$
\varphi(t)=C_{1}+C_{2} \theta \sqrt[3]{4\left(t-\frac{1}{2}\right)}
$$

where $\left(C_{1}, C_{2}\right) \in R^{2}$ is a fixed point of the operator $V_{k}$ (3.1).

Proof. Necessariness. Let $\varphi \in C[0,1]$ be a solution of the equation (3.2). Then we have

$$
\varphi(t)=C_{1}+C_{2} \theta \sqrt[3]{4\left(t-\frac{1}{2}\right)},
$$

where

$$
\begin{gathered}
C_{1}=\int_{0}^{1} \varphi^{k}(u) d u \\
C_{2}=\int_{0}^{1} \sqrt[3]{\left(u-\frac{1}{2}\right)} \varphi^{k}(u) d u
\end{gathered}
$$

Substituting function $\varphi(t)$ (3.3) into (3.4) we obtain

$$
C_{1}=3\left(\frac{\left(C_{1}+C_{2} \theta \sqrt[3]{2}\right)^{k+1}-\left(C_{1}-C_{2} \theta \sqrt[3]{2}\right)^{k+1}}{2 \sqrt[3]{2}(k+1) C_{2} \theta}-\frac{\left(C_{1}+C_{2} \theta \sqrt[3]{2}\right)^{k+2}+\left(C_{1}-C_{2} \theta \sqrt[3]{2}\right)^{k+2}}{\sqrt[3]{4}(k+1)(k+2) C_{2}^{2} \theta^{2}}+\right.
$$




$$
\left.+\frac{\left(C_{1}+C_{2} \theta \sqrt[3]{2}\right)^{k+3}-\left(C_{1}-C_{2} \theta \sqrt[3]{2}\right)^{k+3}}{2(k+1)(k+2)(k+3) C_{2}^{3} \theta^{3}}\right) .
$$

Substituting the function $\varphi(t)(3.3)$ into (3.5) we get

$$
\begin{aligned}
& C_{2}=3\left(\frac{\left(C_{1}+C_{2} \theta \sqrt[3]{2}\right)^{k+1}+\left(C_{1}-C_{2} \theta \sqrt[3]{2}\right)^{k+1}}{2 \sqrt[3]{4}(k+1) C_{2} \theta}-\frac{3\left(\left(C_{1}+C_{2} \theta \sqrt[3]{2}\right)^{k+2}-\left(C_{1}-C_{2} \theta \sqrt[3]{2}\right)^{k+2}\right)}{4(k+1)(k+2) C_{2}^{2} \theta^{2}}+\right. \\
& \left.+\frac{3\left(\left(C_{1}+C_{2} \theta \sqrt[3]{2}\right)^{k+3}+\left(C_{1}-C_{2} \theta \sqrt[3]{2}\right)^{k+3}\right)}{2 \sqrt[3]{2}(k+1)(k+2)(k+3) C_{2}^{3} \theta^{3}}-\frac{3\left(\left(C_{1}+C_{2} \theta \sqrt[3]{2}\right)^{k+4}-\left(C_{1}-C_{2} \theta \sqrt[3]{2}\right)^{k+4}\right)}{2 \sqrt[3]{4}(k+1)(k+2)(k+3)(k+4) C_{2}^{4} \theta^{4}}\right) .
\end{aligned}
$$

Consequently the point $\left(C_{1}, C_{2}\right) \in R^{2}$ is a fixed point of the operator $V_{k}(3.1)$.

Sufficiency. Suppose that a point $\left(C_{1}, C_{2}\right) \in R^{2}$ is a fixed point of the operator $V_{k}$ define the function $\varphi(t) \in C[0,1]$ by the equality

$$
\varphi(t)=C_{1}+C_{2} \theta \sqrt[3]{4\left(t-\frac{1}{2}\right)}
$$

Then

$$
\begin{aligned}
& \left(H_{k} \varphi\right)(t)=\int_{0}^{1}\left(1+\sqrt[3]{4} \theta \sqrt[3]{\left(t-\frac{1}{2}\right)\left(u-\frac{1}{2}\right)}\right) \varphi^{k}(u) d u=\int_{0}^{1} \varphi^{k}(u) d u+ \\
& +\sqrt[3]{4} \theta \sqrt[3]{t-\frac{1}{2}} \int_{0}^{1} \sqrt[3]{u-\frac{1}{2}} \varphi^{k}(u) d u=\int_{0}^{1}\left(C_{1}+C_{2} \theta \sqrt[3]{4\left(u-\frac{1}{2}\right)}\right)^{k} d u+ \\
& +\sqrt[3]{4} \theta \sqrt[3]{t-\frac{1}{2}} \int_{0}^{1} \sqrt[3]{u-\frac{1}{2}}\left(C_{1}+C_{2} \theta \sqrt[3]{4\left(u-\frac{1}{2}\right)}\right)^{k} d u= \\
& 3\left(\frac{\left(\alpha+C_{2} \theta \sqrt[3]{2}\right)^{k+1}-\left(C_{1}-C_{2} \theta \sqrt[3]{2}\right)^{k+1}}{2 \sqrt[3]{2}(k+1) C_{2} \theta}-\frac{\left(C_{1}+C_{2} \theta \sqrt[3]{2}\right)^{k+2}+\left(C_{1}-C_{2} \theta \sqrt[3]{2}\right)^{k+2}}{\sqrt[3]{4}(k+1)(k+2) C_{2}^{2} \theta^{2}}+\right. \\
& \left.+\frac{\left(C_{1}+C_{2} \theta \sqrt[3]{2}\right)^{k+3}-\left(C_{1}-C_{2} \theta \sqrt[3]{2}\right)^{k+3}}{2(k+1)(k+2)(k+3) C_{2}^{3} \theta^{3}}\right)+3 \sqrt[3]{4} \theta \sqrt[3]{t-\frac{1}{2}} \times \\
& \times\left(\frac{\left(C_{1}+C_{2} \theta \sqrt[3]{2}\right)^{k+1}+\left(C_{1}-C_{2} \theta \sqrt[3]{2}\right)^{k+1}}{2 \sqrt[3]{4}(k+1) C_{2} \theta}-\frac{3\left(\left(C_{1}+C_{2} \theta \sqrt[3]{2}\right)^{k+2}-\left(C_{1}-C_{2} \theta \sqrt[3]{2}\right)^{k+2}\right)}{4(k+1)(k+2) C_{2}^{2} \theta^{2}}+\right. \\
& \left.\frac{3\left(\left(C_{1}+C_{2} \theta \sqrt[3]{2}\right)^{k+3}+\left(C_{1}-C_{2} \theta \sqrt[3]{2}\right)^{k+3}\right)}{2 \sqrt[3]{2}(k+1)(k+2)(k+3) C_{2}^{3} \theta^{3}}-\frac{3\left(\left(C_{1}+C_{2} \theta \sqrt[3]{2}\right)^{k+4}-\left(C_{1}-C_{2} \theta \sqrt[3]{2}\right)^{k+4}\right)}{2 \sqrt[3]{4}(k+1)(k+2)(k+3)(k+4) C_{2}^{4} \theta^{4}}\right)= \\
& =C_{1}+C_{2} \theta \sqrt[3]{4\left(t-\frac{1}{2}\right)}=\varphi(t),
\end{aligned}
$$

i.e. the function $\varphi(t)$ is a solution of the equation (3.2). 
4. A phase transition for the model (2.1) at $k=2$

For $k=2$ the operator $V_{2}:(x, y) \in \mathbb{R}^{2} \rightarrow\left(x^{\prime}, y^{\prime}\right) \in \mathbb{R}^{2}$ (see (3.1)) has the form

$$
\left\{\begin{array}{c}
x^{\prime}=x^{2}+\frac{3 \sqrt[3]{4}}{5} \theta^{2} y^{2} \\
y^{\prime}=\frac{6}{5} \theta x y
\end{array}\right.
$$

Proposition 4.1. a) If $0 \leq \theta \leq \frac{5}{6}$, then the Hammerstein's operator $\mathrm{H}_{2}$ has unique (nontrivial) positive fixed point in the $C[0,1]$.

b) If $\frac{5}{6}<\theta<1$, then there are exactly three positive fixed points in $C[0,1]$ of the Hammerstein's operator.

Proof. Clearly, that in the case $\theta=0$ the Hammerstein's operator $H_{2}$ has unique nontrivial positive fixed point $\varphi(t) \equiv 1$. Let $\theta \neq 0$. We consider the system of equations for a fixed point of the operator $V_{2}$ :

$$
\left\{\begin{array}{c}
x^{2}+\frac{3 \sqrt[3]{4}}{5} \theta^{2} y^{2}=x \\
\frac{6}{5} \theta x y=y
\end{array}\right.
$$

In the case $y=0$ from (4.2) we have two solutions $(0 ; 0)$ and $(1 ; 0)$. By proposition 3.1 functions

$$
\varphi(t)=\varphi_{0}(t) \equiv 0, \quad \varphi(t)=\varphi_{1}(t) \equiv 1
$$

are solutions of the equation (4.2).

Suppose $y \neq 0$ in the (4.2). Then from (4.2) we get $x=\frac{5}{6 \theta}$. Consequently, from the first equation of (4.6) we get

$$
y^{2}=\frac{25}{3 \sqrt[3]{4} \theta^{2}} \cdot \frac{6 \theta-5}{36 \theta^{2}} .
$$

Hence it follows, that $\theta>\frac{5}{6}$ and

$$
y=y_{1}^{ \pm}= \pm \frac{5}{6 \theta^{2}} \cdot \frac{1}{\sqrt[3]{2}} \cdot \sqrt{\frac{6 \theta-5}{3}} .
$$

Thus, in the case $0 \leq \theta \leq \frac{5}{6}$ operator $V_{2}$ has two fixed points: $(0 ; 0)$, (1;0) and in the case $\frac{5}{6}<\theta<1$ the operator $V_{2}$ has four fixed points: $(0 ; 0),(1 ; 0),\left(x_{1}, y_{1}^{+}\right)$and $\left(x_{1}, y_{1}^{-}\right)$, with $x_{1}=\frac{5}{6 \theta}$.

Note that, there is no any other fixed point for $V_{2}$.

Consequently,

$$
\begin{gathered}
\varphi_{1}(t) \equiv 1, \\
\varphi_{2}(t)=\frac{5}{6 \theta}\left(1+\sqrt{\frac{6 \theta-5}{3}} \cdot \sqrt[3]{2\left(t-\frac{1}{2}\right)}\right), \\
\varphi_{3}(t)=\frac{5}{6 \theta}\left(1-\sqrt{\frac{6 \theta-5}{3}} \cdot \sqrt[3]{2\left(t-\frac{1}{2}\right)}\right)
\end{gathered}
$$


are non trivial fixed points of the Hammerstein's operator $H_{2}$. Thus we have proved the following

Theorem 4.2. a) If $0 \leq \theta \leq \frac{5}{6}$, then for the model (2.1) on the Cayley tree $\Gamma^{2}$ there exists a unique translational - invariant Gibbs measure;

b) If $\frac{5}{6}<\theta<1$, then for the model (2.1) on the Cayley tree $\Gamma^{2}$ there are three translational - invariant Gibbs measures.

5. A phase transition for the model (2.1) at $k=3$

For $k=3$ the operator $V_{3}:(x, y) \in \mathbb{R}^{2} \rightarrow\left(x^{\prime}, y^{\prime}\right) \in \mathbb{R}^{2}$ (see (3.1)) has the form

$$
\left\{\begin{array}{c}
x^{\prime}=x^{3}+\frac{18}{5} \cdot \frac{\theta^{2}}{\sqrt[3]{2}} x y^{2} \\
y^{\prime}=\frac{9}{5} \theta x^{2} y+\frac{6}{7} \cdot \frac{\theta^{3}}{\sqrt[3]{2}} y^{3} .
\end{array}\right.
$$

Proposition 5.1. a) If $0 \leq \theta \leq \frac{5}{9}$, then (in the $C[0,1]$ ) Hammerstein's operator $\mathrm{H}_{3}$ has a unique nontrivial positive fixed point;

b) If $\frac{5}{9}<\theta<1$, then there are exactly three positive fixed points of the Hammerstein's operator $H_{3} C[0,1]$.

Proof. Clearly, that in the case $\theta=0$ operator $H_{3}$ has unique positive fixed point $\varphi(t) \equiv$ 1. Let $\theta \neq 0$. We consider

$$
\left\{\begin{array}{c}
x^{3}+\frac{18}{5} \cdot \frac{\theta^{2}}{\sqrt[3]{2}} x y^{2}=x \\
\frac{9}{5} \theta x^{2} y+\frac{6}{7} \cdot \frac{\theta^{3}}{\sqrt[3]{2}} y^{3}=y .
\end{array}\right.
$$

For $y=0$ from (5.2) we have three solutions of the system equations (5.2): $(0 ; 0)$, $(-1 ; 0)$ and $(1 ; 0)$. By proposition 3.1 functions

$$
\varphi(t)=\varphi_{0}(t) \equiv 0, \varphi(t)=\varphi_{1}^{ \pm}(t) \equiv \pm 1
$$

are solutions of the equation $H_{3} f=f$. For $x=0$ from (5.2) we get three solutions $(0 ; 0),\left(0 ; y_{1}^{+}\right),\left(0 ; y_{1}^{-}\right)$, where

$$
y_{1}^{ \pm}= \pm \frac{\sqrt[6]{2}}{\theta} \cdot \sqrt{\frac{7}{6 \theta}}
$$

Hance the functions

$$
\varphi_{2}^{ \pm}(t)= \pm \sqrt[6]{2} \cdot \sqrt{\frac{7}{6 \theta}} \cdot \sqrt[3]{4\left(t-\frac{1}{2}\right)}
$$

are solutions of the equation $H_{3} f=f$.

Suppose that $x \neq 0$ and $y \neq 0$ in the (5.2). Then the system of equations (5.2) can be rewritten as

$$
\left\{\begin{array}{c}
x^{2}+\frac{18}{5} \cdot \frac{\theta^{2}}{\sqrt[3]{2}} y^{2}=1 \\
\frac{9}{5} \theta x^{2}+\frac{6}{7} \cdot \frac{\theta^{3}}{\sqrt[3]{2}} y^{2}=1
\end{array}\right.
$$


Hence it follows

and

$$
x^{2}=1-\frac{18}{5} \cdot \frac{\theta^{2}}{\sqrt[3]{2}} y^{2}
$$

$$
\frac{9}{5} \theta\left(1-\frac{18}{5} \cdot \frac{\theta^{2}}{\sqrt[3]{2}} y^{2}\right)+\frac{6}{7} \cdot \frac{\theta^{3}}{\sqrt[3]{2}} y^{2}=1
$$

Then

Therefore $\theta>\frac{5}{9}$ and

$$
y^{2}=\frac{105}{164} \cdot \frac{\sqrt[3]{2}}{2 \theta^{2}} \cdot \frac{9 \theta-5}{9 \theta} .
$$

Consequently

$$
y=y_{2}^{ \pm}= \pm \sqrt{\frac{105}{164}} \cdot \frac{1}{\theta \sqrt[3]{2}} \cdot \sqrt{\frac{9 \theta-5}{9 \theta}}
$$

Hence we conclude that

$$
x^{2}=1-\frac{21}{164} \cdot \frac{9 \theta-5}{\theta} \text {. }
$$

$$
x=x_{1}^{ \pm}= \pm \sqrt{1-\frac{21}{164} \cdot \frac{9 \theta-5}{\theta}} .
$$

Thus, the operator $V_{3}(5.1)$ has five fixed points: $(0 ; 0),(-1 ; 0),(1 ; 0),\left(0 ; y_{1}^{+}\right)$and $\left(0 ; y_{1}^{-}\right)$, if $0 \leq \theta \leq \frac{5}{9}$ and $V_{3}$ has nine fixed points: $(0 ; 0),(-1 ; 0),(1 ; 0),\left(0, y_{1}^{+}\right),\left(0 ; y_{1}^{-}\right)$ $\left(x_{1}^{+} ; y_{1}^{+}\right),\left(x_{1}^{+} ; y_{1}^{-}\right),\left(x_{1}^{-} ; y_{1}^{+}\right)$and $\left(x_{1}^{-} ; y_{1}^{-}\right)$, if $\frac{5}{9}<\theta<1$.

Note that the above mentioned solutions are all possible solutions of $V_{3}$.

Consequently by proposition 3.1 the operator $H_{3}$ has unique positive fixed point $\varphi(t)=$ $\varphi_{1}(t) \equiv 1$ if $0 \leq \theta \leq \frac{5}{9}$. In the case $\frac{5}{9}<\theta<1$ the functions

$$
\varphi_{1}(t) \equiv 1, \quad \varphi_{2}(t)=x_{1}^{+}+y_{1}^{+} \theta \sqrt[3]{4\left(t-\frac{1}{2}\right)}, \varphi_{3}(t)=x_{1}^{+}+y_{1}^{-} \theta \sqrt[3]{4\left(t-\frac{1}{2}\right)}
$$

are positive fixed points of the Hammerstein's operator $H_{3}$.

From Proposition 3.1 and Proposition 5.1 it follows that

Theorem 5.2. a) If $0 \leq \theta \leq \frac{5}{9}$ for the model (2.1) on the Cayley tree $\Gamma^{3}$, then there exists a uniqie translational - invariant Gibbs measure;

b) If $\frac{5}{9}<\theta<1$, then there exist there translational - invariant Gibbs measures. 


\section{REFERENCES}

[1] Bleher, P.M. and Ganikhodjaev N.N.: On pure phases of the Ising model on the Bethe lattice. Theor. Probab. Appl. 35 (1990), 216-227.

[2] Bleher, P.M., Ruiz, J. and Zagrebnov V.A.: On the purity of the limiting Gibbs state for the Ising model on the Bethe lattice. Journ. Statist. Phys. 79 (1995), 473-482.

[3] Eshkabilov Yu.Kh, Haydarov F.H., Rozikov U.A.: Uniqueness of Gibbs Measure for Models With Uncountable Set of Spin Values on a Cayley Tree. Math.Phys.Anal.Geom. DOI 10.1007/s11040-0129118-6.

[4] Eshkobilov Yu.Kh., Haydarov F.H., Rozikov U.A.: Non-uniqueness of Gibbs measure for models with uncountable set of spin values on a Cayley tree. Jour. Stat. Phys. (2012), V.147, No.4, p. 779-794.

[5] Ganikhodjaev, N.N.: On pure phases of the ferromagnet Potts with three states on the Bethe lattice of order two. Theor. Math. Phys. 85 (1990), 163-175.

[6] Ganikhodjaev, N.N. and Rozikov, U.A. Description of periodic extreme Gibbs measures of some lattice model on the Cayley tree. Theor. and Math. Phys. 111 (1997), 480-486.

[7] Ganikhodjaev, N.N. and Rozikov, U.A. : The Potts model with countable set of spin values on a Cayley Tree. Letters Math. Phys. 75 (2006), 99-109.

[8] Ganikhodjaev, N.N. and Rozikov, U.A. On Ising model with four competing interactions on Cayley tree. Math. Phys. Anal. Geom. 12 (2009), 141-156.

[9] Preston, C.: Gibbs states on countable sets (Cambridge University Press, London 1974).

[10] Rozikov, U.A. Partition structures of the Cayley tree and applications for describing periodic Gibbs distributions. Theor. and Math. Phys. 112 (1997), 929-933.

[11] Rozikov, U.A. and Eshkabilov, Yu.Kh.: On models with uncountable set of spin values on a Cayley tree: Integral equations. Math. Phys. Anal. Geom. 13 (2010), 275-286.

[12] Sinai,Ya.G.: Theory of phase transitions: Rigorous Results (Pergamon, Oxford, 1982).

[13] Spitzer, F.: Markov random fields on an infinite tree, Ann. Prob. 3 (1975), 387-398.

[14] Suhov, Y.M. and Rozikov, U.A.: A hard - core model on a Cayley tree: an example of a loss network, Queueing Syst. 46 (2004), 197-212.

[15] Zachary, S.: Countable state space Markov random fields and Markov chains on trees, Ann. Prob. 11 (1983), 894-903.

Yu. Kh. Eshrabilov, Faculty of Mechanics and Mathematics National University of

UZBEKISTAN, TASHKENT, UZBEKISTAN.

E-mail address: yusup62@mail.ru

U. A. Rozikov, Institute of mathematics, National University of Uzbekistan, Tashkent, UZBEKISTAN.

E-mail address: rozikovu@yandex.ru

G. I. Botirov, Faculty of Physics and Mathematics of Bukhara State University, Bukhara, UZBEKISTAN.

E-mail address: botirovg@yandex.ru 